\title{
BMC Developmental Biology reviewer acknowledgement 2015
}

Derek Anane

Craig Albertson
USA

Declan W. Ali

Canada

Jeffrey Amack

USA

Ana Angelova Volponi

UK

Christopher Antos

Germany

Michalis Averof

France

Alfred Batschauer

Germany

Nathalie Beaujean

France

Mark Berryman

USA

Marco Biggiogera

Italy

Samuel Breit

Australia

Cathrin Brisken

Switzerland

Aaron Brown

USA

Thomas Carroll

USA

Francesc Cebria

Spain

\author{
Sang-Wook Cha \\ USA
}

Tatiana Chebotareva

UK

Steven Cheng

China

Rosanna Chianese

Italy

Ariel Chipman

Israel

Barry Condron

USA

Frank Conlon

USA

Stephanie Cossette

USA

Selahattin Danisman

Germany

Fiorenza De Bernardi

Italy

Katia Del Rio-Tsonis

USA

Franck Delaunay

France

Alison Dell

USA

Herman Dierick

USA

Virginia Diewert

Canada
Wolfgang Driever

Germany

Richard M. Epand

Canada

Maria-José Escribá

Spain

Alexei Evsikov

USA

Paul Fowler

UK

Tamara Franz-Odendaal

Canada

Brian Gabrielli

Australia

Gary Gaufo

USA

Burhan Gharaibeh

USA

Roman Giger

USA

Sarah Goetz

USA

Kaoru Goto

Japan

Mukesh Kumar Gupta

India

Marnie Halpern

USA

Andreas Heyland

Canada

Correspondence: derek.anane@biomedcentral.com

BioMed Central, Floor 6, 236 Gray's Inn Road, London WC1X 8HB, UK 
Andreas Hiltbrunner

Germany

Seiji Hitoshi

Japan

Katja Hummitzsch

Australia

Helen Irving-Rodgers

Australia

Jr Jacobs

Canada

Anna Jazwinska

Switzerland

Yasuhiko Kawakami

USA

Nobuyuki Kawashima

Japan

Wiliam Kelly

USA

Khandan Keyomarsi

USA

Toshikazu Kondo

Japan

Ping Kong

USA

Laijun Lai

USA

Tilman Lamparter

Germany

Pierre Leclerc

Canada

Armand Leroi

UK

Michael Levin

USA

Gil Levkowitz

Israel

Baojie Li

Singapore

Baojie Li

China

Lingyu Li

USA

Ziyi Li

China
Ching-Ling (Ellen) Lien

USA

Gufa Lin

USA

Susana Lopes

Portugal

Scott Lozanoff

USA

P Mabee

USA

Paul Maddox

USA

Fanny Mann

France

Julio Martin

Spain

Maria D Martin-Bermudo

Spain

Chris Mayhew

USA

Dies Meijer

UK

Myrte Merkestein

UK

Anthony Mescher

USA

Mark Metzstein

USA

Silvia Clotilde Modina Italy

Denise Montell

USA

Bernard Moussian

Germany

David Murphy

UK

Christopher Navara

USA

Xavier Nicol

France

Heiner Niemann

Germany

Hirotaka James Okano Japan
Katsuhiko Ono

Japan

Christopher Ormandy

Australia

Achim Paululat

Germany

Christopher Payne

USA

Natalie Payne

Australia

Francisco Pelegri

USA

Roberta Pennati

Italy

Christian Petersen

USA

M.Dolors Piulachs

Spain

Berenika Anna Plusa

UK

Ken Prehoda

USA

Don Ready

USA

Joao Relvas

Portugal

Rene Rezsohazy

Belgium

Sandra Rieger

USA

Filippo Rijli

Switzerland

Paul Riley

UK

Rakel Romar

Spain

Siegfried Roth

Germany

Charles Sagerstrom

USA

Jean-Luc Scemama USA

Sylvie Schneider-Maunoury France 


$\begin{array}{ll}\text { Norito Shibata } & \text { Piroska Szabo } \\ \text { Japan } & \text { USA } \\ \text { Fraser Sim } & \text { Qinghua Tao } \\ \text { USA } & \text { China } \\ \text { Johan Smitz } & \text { Kevin Temeyer } \\ \text { Belgium } & \text { USA } \\ \text { Josefa Steinhauer } & \text { X Cindy Tian } \\ \text { USA } & \text { USA } \\ \text { Scott Stewart } & \text { Simon Tuck } \\ \text { USA } & \text { Sweden } \\ \text { Michael Stock } & \text { Alexander Vaiserman } \\ \text { Germany } & \text { Ukraine } \\ \text { Padraig Strappe } & \text { Michael Todd Valerius } \\ \text { Australia } & \text { USA } \\ \text { Devi Suman } & \text { Srinivasan Vijayaraghavan } \\ \text { USA } & \text { USA } \\ \text { Genlou Sun } & \text { Xin Wang } \\ \text { Canada } & \text { USA } \\ \text { Beat Suter } & \text { Timothy Weil } \\ \text { Switzerland } & \text { UK } \\ \text { Tohru Suzuki } & \text { Gunther Wennemuth } \\ \text { Japan } & \text { Germany } \\ & \end{array}$

Gary Wessel

USA

Gabrielle Wheway

UK

Gregory Wray

USA

Chun-Fang Wu

USA

Xuechun Xia

China

Ting Xie

USA

Yukiko Yamazaki

USA

Gyesoon Yoon

Korea, South

Xin Yuan

USA

Vincenzo Zappavigna

Italy

Meijia Zhang

China 